\title{
Linfoma no Hodgkin laríngeo. Reporte de un caso
}

\author{
Laryngeal non Hodgkin Lymphoma. Case Report
}

Jaime Osorio $\mathbf{M}^{1}$, Marcelo Faraggi A', Felipe Cardemil M .

\begin{abstract}
RESUMEN
Los linfomas de la laringe son infrecuentes, correspondiendo a menos del $1 \%$ de las neoplasias de esta ubicación. La detección temprana puede permitir una mejor atención y eventualmente un mejor pronóstico.

Se presenta un caso de linfoma no Hodgkin supraglótico derecho, correspondiente a una paciente de sexo femenino de 68 años, que consulta por disfonía progresiva de varios meses de evolución que se intensifica durante el último mes. La nasofibroscopía mostró una lesión tumoral que comprometía el repliegue aritenoepiglótico derecho, con mucosa sana. La tomografía axial computarizada objetivó un tumor de hemilaringe derecha de aspecto sólido que se extendía desde la base de la epiglotis hasta la glotis. La resonancia magnética informó un proceso expansivo que comprometía la región supraglótica derecha, de aspecto benigno, sin infiltración. Se extirpó un tumor submucoso en su totalidad por tirotomía media. En el examen histopatológico se observaron elementos compatibles con linfoma no Hodgkin difuso, lo que fue confirmado por técnica de inmunohistoquímica. Se trató con esquema CHOP de quimioterapia. No ha habido recidiva tumoral al $3^{\circ}$ año de seguimiento.

Los linfomas no Hodgkin de laringe son poco frecuentes. Se describen las características y manejo de este tipo de tumores.
\end{abstract}

Palabras clave: Linfoma no Hodgkin, laringe, quimioterapia.

\begin{abstract}
Lymphomas of the larynx are rare, accounting for less than $1 \%$ of neoplasms in this location. Early detection can allow better care, and possibly a better prognosis.

We present a case of a right supraglottic Non-Hodgkin Lymphoma, corresponding to a female patient of 68 years who consulted for hoarseness of several months that progressed during the last month. Fibroscopic evaluation showed a tumor involving the right aryepiglottic fold, without mucosal lesion. Computed tomography showed a solid tumor of the right hemilarynx, that extends from base of epiglottis to glottis. Magnetic resonance showed tumor expansion process that involves the right epiglottic region, of benign appearence, without infiltration. We perform a complete removal of the submucosal tumor externally by a medial laryngeal thyroidotomy. Histopathological examination
\end{abstract}

Servicio de Otorrinolaringología, Hospital Barros Luco, Santiago, Chile. Universidad de Chile. 
showed elements compatible with diffuse Non-Hodgkin Lymphoma, which was confirmed by immunohistochemestry. CHOP chemotherapy was indicated. Currently, patient followed up for 3 years, with no signs of tumor recurrence.

Non-Hodgkin Lymphomas of the larynx are rare. We describe the characteristics and management of these tumors.

Key words: Non-Hodgkin Lymphoma, larynx, chemotherapy.

\section{INTRODUCCIÓN}

Los síndromes linfoproliferativos o linfomas son un grupo muy heterogéneo de neoplasias con diferente histología, inmunofenotipo y alteraciones moleculares, por lo que presentan diferentes manifestaciones clínicas y tratamiento. Los linfomas se pueden presentar con compromiso ganglionar 0 extraganglionar, llegando estos últimos al 25\% de Ios casos ${ }^{1}$. En la región de cabeza y cuello, la mayoría de los linfomas extraganglionares son de tipo linfoma no Hodgkin (LNH), y el sitio comprometido más frecuente las estructuras del anillo de Waldeyer².

En la laringe, los linfomas representan menos del $1 \%$ de las neoplasias malignas, y corresponden a la segunda neoplasia hematopoyética en esta ubicación después del plasmocitoma. Se han reportado menos de 100 casos de linfoma laríngeo primario en la literatura ${ }^{3,4}$. La detección temprana puede permitir un mejor pronóstico dependiendo del tipo histológico que se encuentre, por lo que es importante reconocer su presentación clínica ${ }^{5}$. El objetivo de este artículo es reportar un caso de LNH primario de laringe y discutir su manejo.

\section{DESCRIPCIÓN DEL CASO}

Paciente de sexo femenino de 68 años de edad con antecedentes de hipertensión arterial crónica, artritis reumatoidea en tratamiento con metotrexato, colecistectomía y lobectomía tiroidea izquierda por adenoma. Consultó por disfonía progresiva de un año de evolución, que en las últimas semanas se agudiza y se asocia a disfagia y odinofagia. No presentaba síntomas sistémicos.

Se realizó nasofibroscopía (NFC) que evidenció masa supraglótica derecha que abombaba el repliegue aritenoepiglótico, banda y seno piriforme derechos. Mucosa y cuerda vocal sin lesiones. Se apreciaba desplazamiento de la vía área (Figura 1).
Se realizó tomografía computarizada (TC) de cuello que mostraba un tumor de hemilaringe derecha de aspecto sólido, de 2,7 × 2,3 × 1,7 cm que se extendía desde base de epiglotis hasta glotis (Figura 2). Para complementar el estudio se

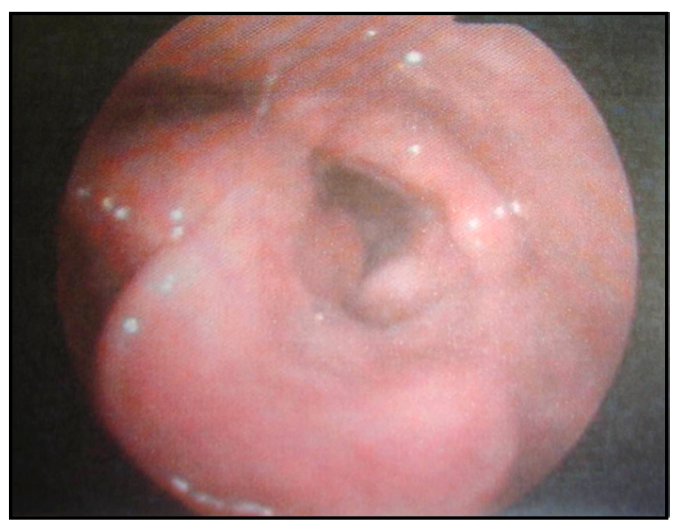

Figura 1. Nasofibroscopía flexible de laringe que evidencia masa supraglótica derecha con mucosa sana, que abomba el repliegue aritenoepiglotico derecho y la banda derecha, la que oblitera el seno piriforme. La imagen está rotada y se aprecia desplazamiento de la vía aérea.

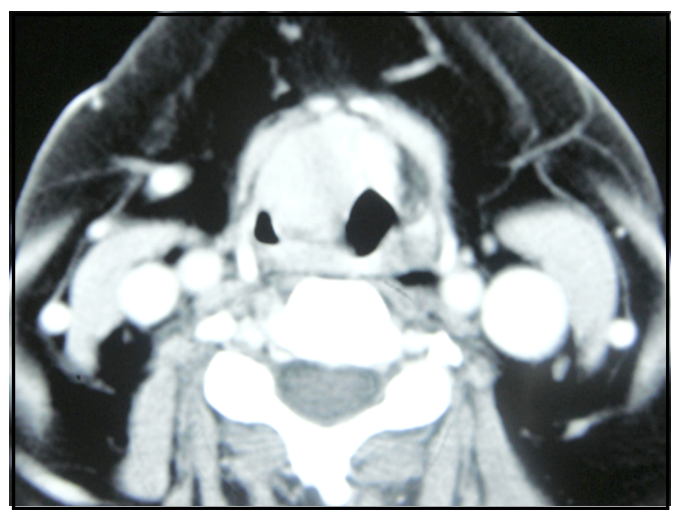

Figura 2. Tomografía computarizada de cuello con contraste endovenoso, imagen de corte axial a nivel supraglótico. Se observa lesión ovoidea que se realza con el medio de contraste en región supraglótica, lateralizada a derecha, obliterando parcialmente la columna aérea. 
realizó resonancia magnética $(\mathrm{RM})$ de cuello, que mostró una lesión en corte axial en T1, con ligera mayor captación de señal que comprometía el vestíbulo laríngeo (Figura 3), y corte sagital en T1 con captación heterogénea de lesión voluminosa que comprometía la región epiglótica derecha, no identificándose compromiso ni invasión categórica de estructuras adyacentes (Figura 4).

Se consideró que se trataba de un tumor benigno submucoso por lo limitado de la lesión,

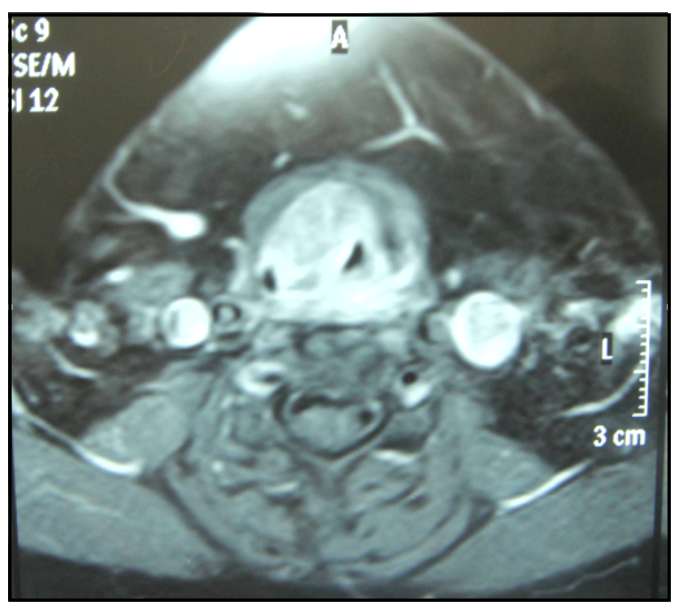

Figura 3. Resonancia magnética de cuello, secuencia T1 axial con uso de gadolinio endovenoso. Se identifica lesión ovoidea supraglótica bien delimitada, observada en tomografía computarizada, que capta gadolinio en forma relativamente homogénea. No se identifica compromiso ni invasión categórica de estructuras adyacentes.

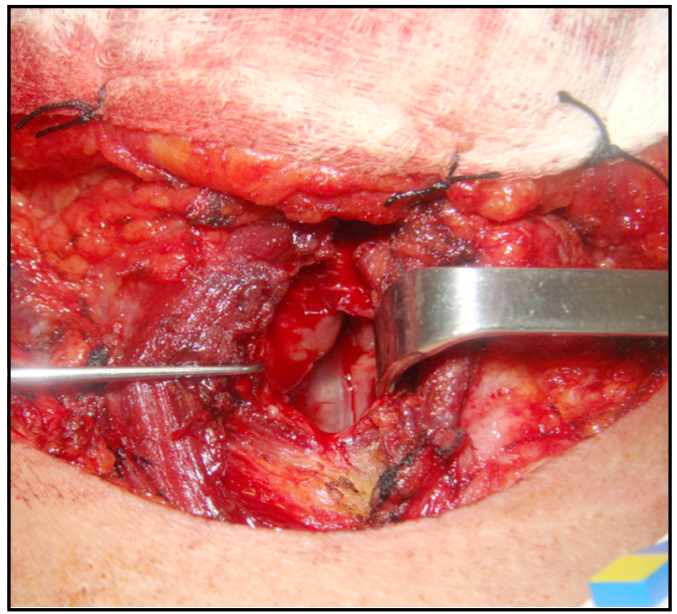

Figura 5. Tirotomía media. Se observa tumor supraglótico derecho. sin infiltración ni diseminación, y considerando la disminución del calibre de la vía aérea, se decidió abordar por tirotomía media (Figura 5), accediendo al plano submucoso, observándose una masa que se extendía desde la región supraglótica derecha hasta espacio paraglótico, de 1,5 × 3,7 cm la cual se extirpó en su totalidad (Figura 6). La paciente presentó buena evolución posoperatoria.

El informe anatomopatológico reveló tres fragmentos irregulares, café pardusco, de 6 gramos,

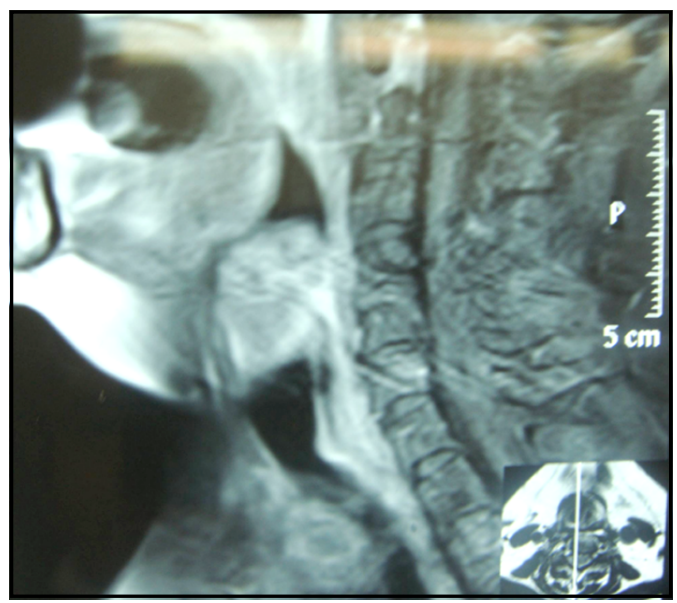

Figura 4. Resonancia magnética de cuello, secuencia T1 sagital con uso de gadolinio endovenoso. Se delimita adecuadamente en sentido céfalo caudal la lesión anteriormente descrita. Destaca su extensión proximal al plano de localización de la epiglotis. Importante disminución del calibre de la columna aérea. No se identifica infiltración de planos adyacentes.

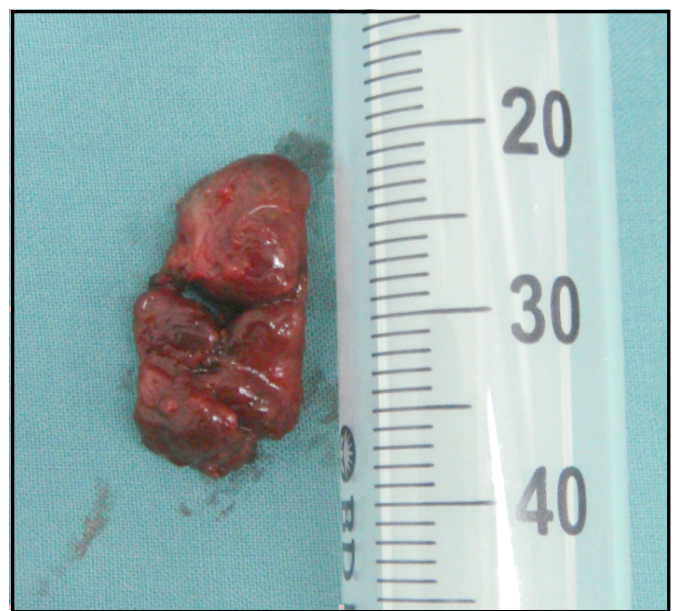

Figura 6. Lesión extirpada, correspondiente a linfoma no Hodgkin laríngeo. 
homogéneos sólidos. Al corte se observaban elementos de neoformación de alta densidad celular con aspecto linfoide, con moderado índice mitótico, sin necrosis, planteándose diagnóstico de linfoma no Hodgkin difuso. Se realiza estudio inmunohistoquímico, que mostró CD20 positivo intenso con patrón nodular, CD10 positivo intenso con disposición nodular, Bcl6 positivo intenso con disposición nodular, MUM-1 positivo patrón nodular, CD3 negativo, bcl2 negativo, CD30 negativo. Por esta razón, se concluye diagnóstico inmunohistoquímico de linfoma folicular mayoritariamente nodular (sobre el $80 \%$ ) grado 3.

Se envía a hematología, quedando etapificado como linfoma folicular estadio IE grado 3 , de acuerdo a la clasificación de Ann Arbor. Se realizaron 6 ciclos de quimioterapia con ciclosfosfamida, doxorubicina, vincristina y prednisolona (CHOP), con remisión completa del tumor. Se realiza NFC de control posterior al tratamiento, como muestran la Figuras 7 y 8, objetivándose la ausencia de lesión en supraglotis, con movilidad cordal normal. No ha habido recidiva tumoral al $3^{\circ}$ año de seguimiento.

\section{DISCUSIÓN}

Entre los LNH primarios de laringe, el subtipo histológico más frecuente corresponde a linfomas B difusos de células grandes, linfoma marginal extranodal, y tejido linfático asociado a mucosa $(\text { MALT })^{6}$. En el presente caso el subtipo fue linfoma folicular de estirpe celular $B$, de los cuales existen escasos reportes en la literatura. Este subtipo de linfoma se relaciona con alteración de los fenómenos de la apoptosis. Aún más raros son los linfomas de células $T$ en laringe ${ }^{2}$. En cualquier caso, debe considerarse que este cuadro es similar a otros linfomas, lo que lo hace particular es su ubicación.

Dentro de los factores que se asocian al desarrollo de LNH en laringe se encuentra el uso de metotrexato en pacientes con artritis reumatoide, como es el caso de esta paciente, pero esta asociación es controversial y no ha sido bien establecida ${ }^{7,8}$. La localización más común de crecimiento de los LNH primarios de laringe es la región supraglótica, ya que contiene mayor tejido linfoide.

Markou y cols realizaron una revisión de la mayoría de los casos notificados en idioma inglés de LNH primario de la laringe desde 1996 hasta 2008 , encontrando que el $47 \%$ de ellos se encuentran en la región supraglótica, el $25 \%$ en el área de la glotis, y el resto subglótico o transglótico². En la serie de Siddiqui y cols, se apreció que el 100\% comprometió la supraglotis, existiendo compromiso de la glotis en $65 \%$ de éstos, de la hipofaringe en $60 \%$, y de la subglotis en $35 \%$ de los $\operatorname{casos}^{9}$. La mayoría de los LNH son inicialmente vistos como una lesión no ulcerada o masas polipoídeas en la epiglotis 0 repliegue aritenoepioglótico. En una serie, de 36 casos, $55 \%$ se describieron como masas lisas o submucosa, mientras que sólo 5,5\% fueron lesiones ulceradas².

La edad de aparición de linfomas laríngeos varía entre 4 y 90 años, con una media edad de aparición de 63 años, y una relación femenino:masculino de 2:19. En el presente caso corresponde a una mujer de 68 años ${ }^{2}$. La sintomatología habitual inicial de los pacientes con LNH primario de laringe incluyen disfonía, tos,

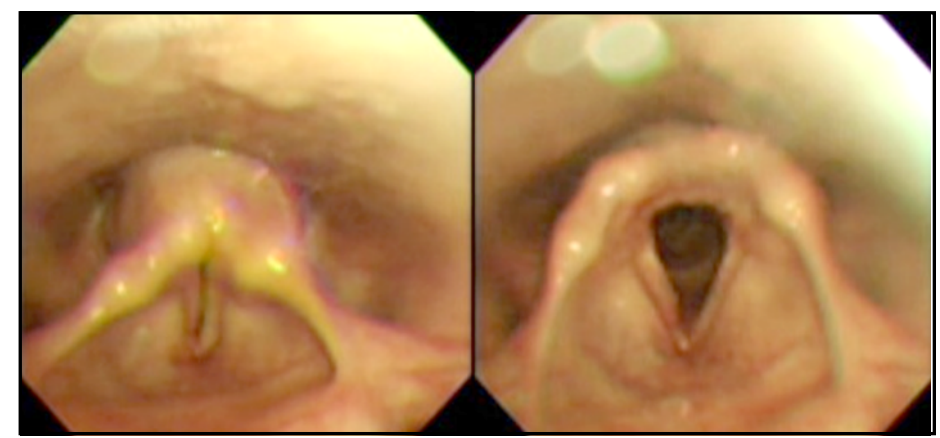

Figura 7 y 8 . Nasofibroscopía flexible de laringe. En control, se objetiva ausencia de lesión supraglótica con movilidad cordal normal. 
disfagia, sensación de cuerpo extraño, estridor 0 signos sistémicos, como pérdida de peso y fiebre. Presentan una duración de los síntomas que varía de 10 días hasta 1 año $0^{5}$. En el caso de esta paciente, no presentó síntomas sistémicos, lo que apoya el argumento de ser una lesión circunscrita a la laringe.

Respecto a las técnicas de imagen, pueden ser útiles en la evaluación de cualquier neoplasia laríngea. Se puede encontrar en la RM señal homogénea intermedia en $\mathrm{T} 1$, variabilidad de intensidad de la señal en T2, e hiperintensidad y reforzamiento del tumor en imágenes en T1 contrastadas con gadolinio y supresión grasa ${ }^{10}$, pero estas características son inespecíficas, por lo que la RM se utiliza principalmente para determinar la extensión del tumor. Se puede apreciar invasión de cartílagos laríngeos 0 adenopatías cervicales en hasta 20\% de los casos. No se observan imágenes de necrosis ni calcificación ${ }^{9}$. A la imagen con TC, se aprecia reforzamiento al contraste yodado ${ }^{10}$. Todos los linfomas laríngeos captarían fludesoxiglucosa (FDG), el medio de captación más utilizado en las imágenes por PET, por lo que este método tendría utilidad en la evaluación ${ }^{9}$. Para el diagnóstico de anatomía patológica, se debe asociar la histología, análisis inmunohistoquímicos, y cada vez se consideran más análisis, como citometría de flujo 0 $\mathrm{FISH}^{6}$. Dentro del diagnóstico diferencial morfológico se debe considerar carcinoma indiferenciado, melanoma, linfoma de Burkitt, y leucemias agudas, lo que será resuelto con la ayuda de la inmunohistoquímica ${ }^{6}$.

La radioterapia y la quimioterapia son las estrategias terapéuticas utilizadas más frecuentemente en todo el mundo para el tratamiento de los linfomas laríngeos primarios. La literatura indica que el linfoma localizado en la laringe es uno de los tumores más radiosensibles de esa localización, pero al ser una enfermedad sistémica, se ha sugerido que el tratamiento sea combinado (radioquimioterapia) o quimioterapia exclusiva, especialmente para la recurrencia 0 diseminación ${ }^{2-}$ ${ }^{5,9,11}$. El esquema de quimioterapia de elección sería el CHOP (ciclofosfamida, doxorubicina, vincristina, y prednisolona) $)^{6}$, a lo que se puede agregar rituximab ${ }^{12}$. El tratamiento de elección no es quirúrgico, sin embargo, la cirugía se puede utilizar cuando se presentan los linfomas con obstrucción de la laringe 0 hemorragia masiva ${ }^{2}$. En el presente caso, se asumió que era un tumor limitado a la laringe con cierto grado de obstrucción de vía aérea, por la sintomatología local, la larga duración de los síntomas y la ausencia de síntomas sistémicos, además de las imágenes que mostraban una lesión delimitada sin infiltración ni captación inespecífica, por lo que se decidió realizar resección completa de la lesión, ya que una biopsia iba ser una resección incompleta lo que aumentaba el riesgo de obstrucción y sangrado. Una vez que se tuvo el diagnostico de linfoma se realizó el tratamiento definitivo con quimioterapia. Nayak y cols reportaron un caso de linfoma laríngeo estudiado extensamente con microlaringoscopía, histología, inmunohistoquímica, citometría de flujo, cariotipificación e imagenología, diagnosticándose el primer caso de linfoma difuso de células $B$ no MALT, que recibió tratamiento con quimioterapia e inmunoterapia específica, lo que refuerza el concepto de obtención de una cantidad de tejido diagnóstico adecuado en tumores laríngeos de presentación poco habitual'11.

Respecto al pronóstico, la sobrevida considerando todos los estadios se reporta de entre $40 \%$ a $70 \%{ }^{12}$, sin embargo en estadios tempranos los linfomas foliculares presentan tasas de curación en torno al $95 \%$, la que disminuye a menos del $50 \%$ en estadios avanzados ${ }^{1,5}$.

\section{CONCLUSIÓN}

En conclusión, el linfoma de laringe primario es poco frecuente, y se debe considerar en el diagnóstico diferencial en tumores de laringe de larga data con mucosa sana, especialmente en el área supraglótica. El linfoma primario de laringe es una inusual presentación de los LNH, y deben ser manejados de acuerdo a las tendencias actuales del tratamiento de los LNH.

\section{BIBLIOGRAFÍA}

1. SuÁREZ C. Tratado de otorrinolaringología y cirugía de cabeza y cuello. 2009 Tomo IV Cirugía oncológica de cabeza y cuello y de la base del cráneo. Síndromes linfoproliferativos en el área otorrinolaringológica. 4037- 4049. 
2. Markou K, Goudakos J, Constantinidis J, Kostopoulos I, Vital V, NikolaOu A. Primary laryngeal lymphoma: report of 3 cases and review of the literature. Head Neck 2010; 32(4): 541-9.

3. Word R, Urquhart aC, Ejercito VS. Primary laryngeal lymphoma case report. Ear Nose Throat J 2006; 85(2): 109-11.

4. Andriychuk A, Kristensen BW. Non-Hodgkin lymphoma of the larynx. Ugeskr Laeger 2010; 172(42): 2901-2.

5. Franzen A, Kurrer MO. Malignant Lymphoma of the Larynx: Case Report and Review of the Literature. Laryngorhinootologie 2000; 79(10): 579-83.

6. Desal SC, Allen C, Chernock R, Haughey B. Pathology Quiz Case 1: Primary diffuse large Bcell lymphoma of the larynx. Arch Otolaryngol Head Neck Surg FALTA AÑO; 137 (5): 526-528.

7. Patiar S, Ramsden JD, Freeland AP. B-cell Iymphoma of the larynx in a patient with rheumatoid arthritis. J Laryngol Otol 2005; 119(8): 646-8.
8. Acero J, Navarro-Cuellar C, Menarguez J, Herencia $\mathrm{H}$, NavarRo-VILA C. Naso-maxillary non-Hodgkin lymphoma associated with methotrexate treatment in a patient with rheumatoid arthritis. J Oral Maxillofac Surg 2006; 64(4): 708-11.

9. Siddiqui NA, Branstetter BF, Hamilton BE, Ginsberg LE, Glastonbury CM, Harnsberger HR, et al. Imaging characteristics of primary laryngeal lymphoma. AJNR Am J Neuroradio/2010; 31(7): 1261-5.

10. Takayama F, Takashima $S$, Momose M, Arakawa K, MIYASHITA K, SONE S. MR imaging of primary malignant lymphoma in the larynx. Eur Radiol 2001; 11(6): 1079-82.

11. Nayak JV, Cook JR, Molina JT, Branch MP, Branstetter BF, Ferris RL, et al. Primary lymphoma of the larynx: new diagnostic and therapeutic approaches. J Otorhinolaryngol Relat Spec 2003; 65(6): 321-6.

12. BRusamolino E. First-line therapy of CD20 diffuse large B-cell lymphoma: facts and open questions. Haematologica 2009; 94(9): 1194-8.

Dirección: Jaime Osorio Mallea Hospital Barros Luco Gran Avenida José Miguel Carrera 3204, San Miguel Teléfono: 02-5763000

E mail: jaime_osorio@yahoo.com 